\title{
COVID-19 - rheumatic diseases and rheumatologists
}

\author{
Maria Maślińska ID
}

Early Arthritis Clinic, National Institute of Geriatrics, Rheumatology and Rehabilitation, Warsaw, Poland

After battling-for more than a year - the SARS-CoV-2 pandemic and its successive waves affecting different regions of the world, the medical community, regardless of the specialization its members represent, has achieved a basic understanding of the main problems this virus poses. The knowledge gained throughout the pandemic concerns a wide range of issues - from the course of the disease, which is often unpredictable, through the methods of therapeutic treatment, to the emergence of the post-COVID phenomena.

Since the emergence of the pandemic one of the fundamental problems encountered by the medical community has been the identification of factors that negatively influence the outcome of the infection and those that seem beneficial for the patients.

Initially, the age of patients appeared to be partic ularly relevant in this context, though that conclusion has gradually changed, as the symptomatic infection has begun to affect increasingly younger individuals. During the recent "third wave" of the pandemic in Poland, the symptomatic infection affected mostly people aged 40-60, more often men; these data correlated with the experience of other European countries [1]. The prognosis of the negative outcome of the infection depended to a large extent on the concurrent presence of diseases associated with the metabolic syndrome. Still, the rapid and severe course of the disease has also been observed in younger patients without any significant risk factors identified so far.

The question still remains, how do rheumatic diseases (RD) influence the clinical picture of a COVID-19 infection? Does an autoimmune disease, often resulting in immunosuppressive therapy or a predominantly anticytokine biological treatment, e.g. in rheumatoid arthritis, have a negative impact on the course of COVID-19?

Data available from the COVID-19 Global Rheumatology Alliance physician registry (C19-GRA) reveal that of the 1,324 studied RD patients $36 \%$ were hospitalized and $6 \%$ died [2]. The use of mechanical ventilation was necessary in $26 \%$ of cases. Data from the United States indicated that African American patients, Latinx patients and Asian patients ( $O R=2.74,1.71,2.69$ respectively) had in multivariable models higher odds of hospitalization compared to White patients, with no differences between ethnicities in mortality being revealed [2, 3]. These findings are still to be verified, as all the registries are voluntary, which may result in errors in patient selection and incomplete data entry (it does not cover all rheumatic disease patients with COVID-19 in the observed population) as well as the incorrect assignment of race/ethnicity by a describing physician [2-4].

As in states such as Poland, with a more homogeneous ethnic structure, reliable comparisons in this respect are not possible, the need for creation of international registers and for cooperation within their structures becomes evident.

Among all patients in the National Institute of Geriatrics, Rheumatology and Rehabilitation hospitalized due to the COVID-19 infection during the "third wave" of the pandemic in Poland (March-May 2021), only about $2 \%$ had inflammatory rheumatic diseases among revealed comorbidities and no patients from this group died during the hospitalization. What is important, the presence of the RD had no essential impact on the course of infection, with other comorbidities - such as diabetes, chronic obstructive pulmonary disease (COPD) or coronary artery disease with chronic circulatory failure - playing a vital role in this respect.

The COVID-19 pandemic also brought unintended opportunities associated with the diagnostic process undertaken during the infection. An example of such a situation is the discovery of aortic aneurysm lesions in hospitalized COVID-19 patients, as computed tomography (CT) scans of the chest were performed in the majority of this group [5]. These findings, associated with poorly controlled arterial pressure and the progressive atherosclerotic process, were mostly asymptomatic or scarcely symptomatic, and only the diagnostic pro-

Address for correspondence:

Maria Maślińska, Early Arthritis Clinic, National Institute of Geriatrics, Rheumatology and Rehabilitation, 1 Spartanska St., 02-637 Warsaw, Poland, e-mail: maslinskam@gmail.com

Submitted: 15.06.2021; Accepted: 21.06.2021 


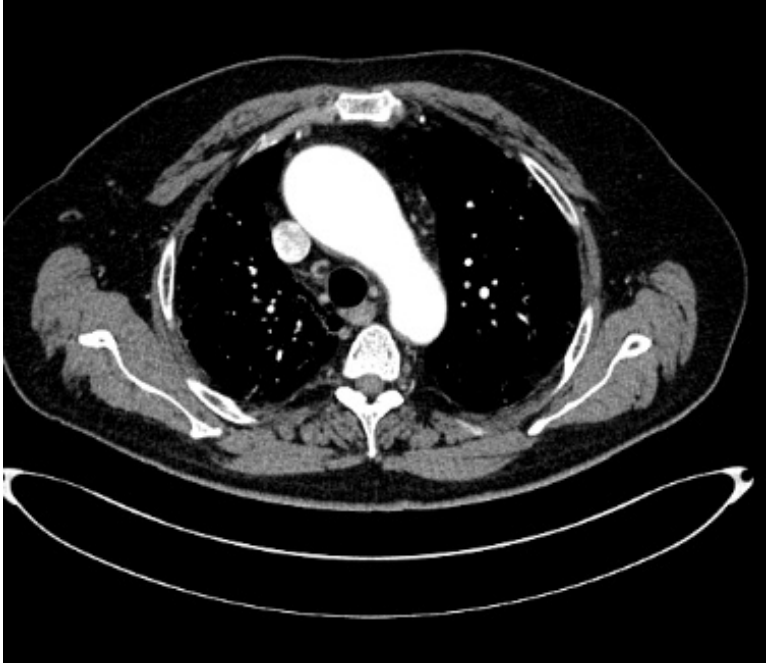

Fig. 1. A newly detected aortic aneurysm in a patient with pneumonia in the course of COVID-19 (the photo was taken in the Department of Radiology, National Institute of Geriatrics, Rheumatology and Rehabilitation).

cess associated with the infection drew attention to the existing, yet unrecognized complex problem. The wide use of CT chest scans also enabled the analysis of the progress of aneurysms in patients who had this problem diagnosed previously.

Figure 1 presents a newly detected aortic aneurysm in a patient with pneumonia in the course of COVID-19.

According to other recently presented observations and studies concerning the course of COVID-19, there is a significant increase in clotting system activation markers such as D-dimers, international normalized ra- tio (INR) or activated partial thromboplastin time (APTT) prolongation, with or without overlapping thromboembolic changes in the pulmonary and extrapulmonary vessels [6]. These phenomena were also observed in our hospitalized patients. Figure 2 shows a pulmonary embolism confirmed with CT.

However, the increase in D-dimers should also be related to the activity of the inflammatory process, which automatically translates into an increase in the concentrations of other acute phase proteins, e.g. fibrinogen and alkaline phosphatase. Among all parameters of inflammation, hyperferritinemia was most strongly associated with the intensification of pulmonary lesions, which was demonstrated in our pilot study of 39 patients $(p<0.0001)$. Interestingly, our preliminary data do not indicate that the blood saturation on patient's admission correlates with the area measured of lung involvement assessed in the CT examination in this study group.

We observed, confirming numerous similar reports of other clinicians battling COVID-19, different post-COVID symptoms, such as Raynaud's phenomenon, vasculitides (often small vessels, skin vessels) and symptoms of fibromyalgia [7]. Further observations are required to determine whether and how the current pandemic will affect the picture of previously confirmed autoimmune diseases, including the progress of organ changes, especially affecting the lung tissue, and how the SARS-CoV-2 infection will affect the de novo development of autoimmune diseases, including rheumatic diseases. What is especially noteworthy is the occurrence of chronic fatigue and often cognitive disorders, referred to as "brain fog", in individuals long after the acute infection phase [8].
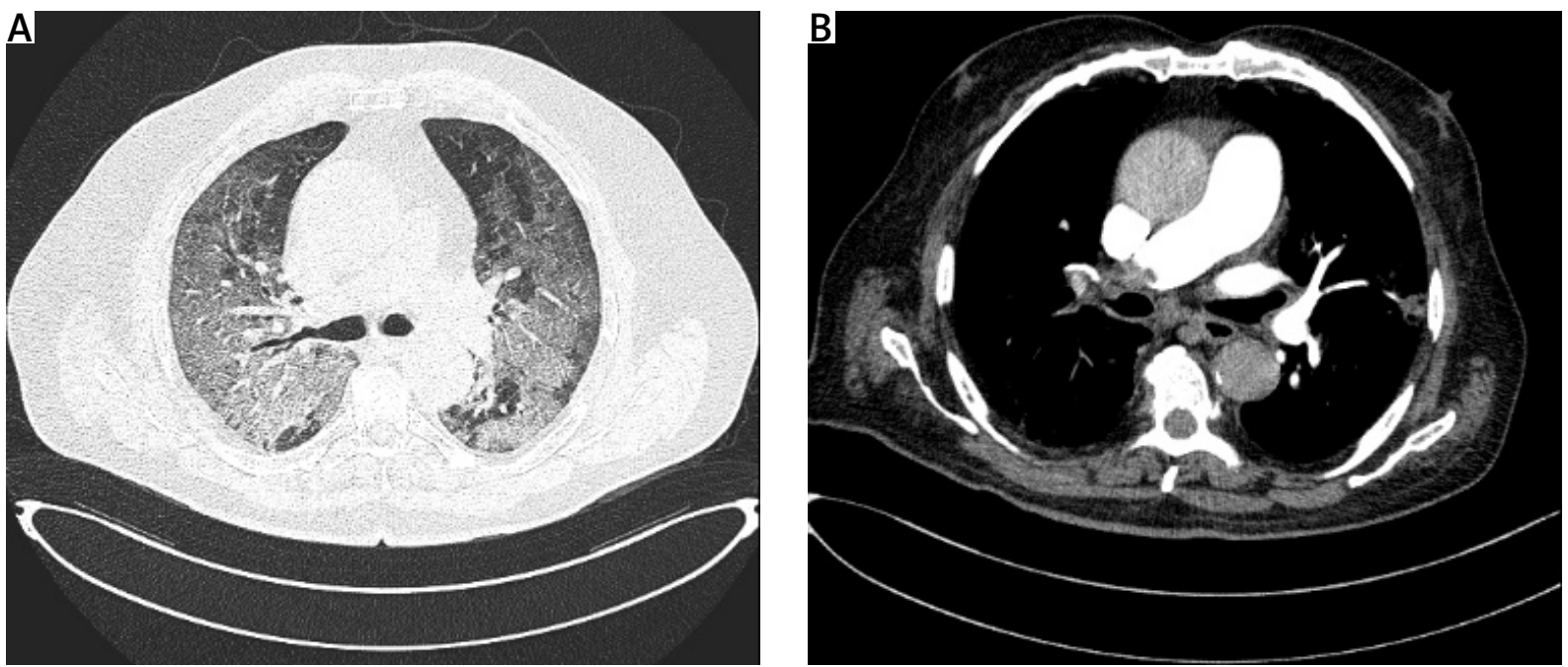

Fig. 2. A pulmonary embolism confirmed with computed tomography (the photos were taken in the Department of Radiology, National Institute of Geriatrics, Rheumatology and Rehabilitation). 
These symptoms are also often present in systemic connective tissue diseases such as Sjögren's syndrome or systemic lupus erythematosus and should not be ignored. The question remains how to introduce effective evaluation mechanisms, e.g. assessing the severity of chronic fatigue or brain fog, in everyday medical practice. It seems that for rheumatologists the coming years bring the prospect of closer cooperation with other specialists, such as pulmonologists, cardiologists, psychologists and psychiatrists.

A separate problem that requires description and analysis is the management of the stress the medical personnel in different hospitals and of different specializations has to cope with at the time of an epidemiological catastrophe $[9,10]$. This issue concerns dealing with the extreme doctor-patient, doctor-patient's family and interpersonal relations in times of maximal and prolonged stress. From the experience of our team, immediate psychological help turned out to be both very necessary and effective. The question remains as to the long-term negative and positive consequences of this situation.

\section{Acknowledgements}

I would like to thank all my colleagues from the Early Arthritis Clinic and the Department of Systemic Connective Tissue Diseases, National Institute of Geriatrics, Rheumatology and Rehabilitation and all other medical workers (anesthesiologists, radiologists, psychologist, physiotherapists, technicians) who worked during the third wave of the COVID-19 pandemic in our COVID 3 department.

The author declares no conflict of interests.

\section{References}

1. Fisayo T, Tsukagoshi S. Three waves of the COVID-19 pandemic. Postgrad Med J 2021; 97: 332, DOI: 10.1136/postgradmedj-2020-138564.

2. Gianfrancesco MA, Leykina LA, Izadi Z, et al. Association of race and ethnicity with COVID-19 outcomes in rheumatic disease: data from the COVID-19 Global Rheumatology Alliance Physician Registry. Arthritis Rheumatol 2021; 73: 374-380, DOI: 10.1002/art.41567.

3. Hyrich KL, Machado PM. Rheumatic disease and COVID-19: epidemiology and outcomes. Nat Rev Rheumatol 2021; 17: 71-72, DOI: 10.1038/s41584-020-00562-2.

4. Strangfeld A, Schäfer M, Gianfrancesco MA, COVID-19 Global Rheumatology Alliance, et al. Factors associated with COVID-19-related death in people with rheumatic diseases: results from the COVID-19 Global Rheumatology Alliance physician-reported registry. Ann Rheum Dis 2021; 80: 930-942, DOI: 10.1136/annrheumdis-2020-219498.

5. Wang J, Saguner AM, An J, et al. Dysfunctional coagulation in COVID-19: from cell to bedside. Adv Ther 2020; 7: 3033-3039, DOI: 10.1007/s12325-020-01399-7.

6. Griffin CL, Sharma V, Sarfati MR, et al. Aortic disease in the time of COVID-19 and repercussions on patient care at an academic aortic center. J Vasc Surg 2020; 72: 408-413, DOI: 10.1016/j.jvs.2020.04.487.

7. Cañas CA. The triggering of post-COVID-19 autoimmunity phenomena could be associated with both transient immunosuppression and an inappropriate form of immune reconstitution in susceptible individuals. Med Hypotheses 2020; 145: 110345, DOI: 10.1016/j.mehy.2020.110345.

8. Salamanna F, Veronesi F, Martini L, et al. Post-COVID-19 syndrome: the persistent symptoms at the post-viral stage of the disease. A systematic review of the current data. Front Med (Lausanne) 2021; 8: 653516, DOI: 10.3389/fmed.2021.653516.

9. Wu W, Zhang Y, Wang P, et al. Psychological stress of medical staffs during outbreak of COVID-19 and adjustment strategy. J Med Virol 2020; 92: 1962-1970, DOI: 10.1002/jmv.25914.

10. Lima CKT, Carvalho PMM, Lima IAAS, et al. The emotional impact of coronavirus 2019-Ncov (new coronavirus disease). Psychiatry Res 2020; 287: 112915, DOI: 10.1016/j.psychres. 2020.112915. 